\title{
Superconducting Exchange Coupling between Ferromagnets
}

\author{
Yi Zhu, Avradeep Pal, Mark G. Blamire*, Zoe H. Barber
}

Recent discoveries from superconductor (S)/ ferromagnet (FM) heterostructures include $\pi$ junctions ${ }^{1}$, triplet pairing ${ }^{2,3}$, critical temperature $\left(T_{c}\right)$ control in FM/S/FM superconducting spin valves (SSVs) ${ }^{4-7}$, and critical current control in S/FM/N/FM/S spin valve Josephson junctions ${ }^{8,9}$ (N: normal metal). In all cases, the magnetic state of the device, generally set by the applied field, controls the superconducting response. We report here the observation of the converse effect, i.e. direct superconducting control of the magnetic state in $\mathrm{GdN} / \mathrm{Nb} / \mathrm{GdN}$ SSVs. A model ${ }^{10}$ for an antiferromagnetic effective exchange interaction based on the coupling of the superconducting condensation energy to the magnetic state can explain the $\mathrm{Nb}$ thickness and temperature dependence of this effect. This superconducting exchange interaction is fundamentally different in origin from the various exchange coupling phenomena which underlie conventional spin electronics (spintronics), and provides a mechanism for the active control of the magnetic state in superconducting spintronics ${ }^{11}$.

Electron exchange coupling between magnetic layers defines the ground state configuration of magnetic heterostructures. In conventional spintronics, an applied field or current-driven spin transfer torque ${ }^{12}$ is used to change the magnetic state - e.g. to write to a memory element. The primary mechanisms of this exchange coupling are direct (Heisenberg) coupling between a ferromagnet (FM) and an antiferromagnet (AFM) to generate exchange bias $^{13}$, and indirect Ruddermann-Kittel-Kasuya-Yoshida (RKKY) coupling ${ }^{14}$ which, for certain non-magnetic $(\mathrm{N})$ spacer thicknesses, can be antiferromagnetic $(\mathrm{AF})$ - crucial to the original discovery of giant magnetoresistance ${ }^{15,16}$ and now widely applied to create synthetic $\mathrm{AFMs}^{17}$. The magnitude of such exchange coupling is controlled by the materials and layer thicknesses, and creates a passive ground state against which active methods of magnetization control must act. Here, we present evidence for a fundamentally different exchange interaction mediated by the superconducting condensation energy and driven by an exceptionally large change in $T_{c}\left(\Delta T_{c}\right)$ between parallel (P) and antiparallel (AP) configurations.

A series of $\operatorname{GdN}(3 \mathrm{~nm}) / \mathrm{Nb}\left(t_{S}\right) / \mathrm{GdN}(5 \mathrm{~nm})$ trilayer superconducting pseudo spin valves were deposited with different $\mathrm{Nb}$ layer thicknesses $t_{S}$ (see Methods). A typical set of resistance versus magnetic field $(R(H))$ curves (Fig. 1a) shows a well-defined infinite magnetoresistance $\left(M R=\left(R_{\max }-R_{\min }\right) / R_{\min }\right)$ with sharp switching between normal and superconducting states. The behaviour is similar to that reported for EuS/A1/EuS devices by Li et al. ${ }^{5}$, but the higher $T_{c}$ of $\mathrm{Nb}$ means that it is observable up to $\sim 6 \mathrm{~K}$ (see right inset to Fig. 2, Supplementary Information 1 and Supplementary Fig. 1) rather than below $T_{c \mathrm{cl}}(\sim 1.5$ $\mathrm{K})$.

Department of Materials Science and Metallurgy, University of Cambridge, 27 Charles Babbage Road, Cambridge CB3 0FS, UK. *e-mail:mb52@cam.ac.uk 




Figure 1| Temperature dependence of spin valve effects in a $\mathrm{GdN}(3) / \mathrm{Nb}(8) / \mathrm{GdN}(5)$ heterostructure. a, Resistance as a function of applied magnetic field $(R(H))$ at different temperatures (see Methods); inset figures show the expected magnetic configurations. $\mathbf{b}$, Magnetization hysteresis loop $(\mathrm{M}(\mathrm{H})$ ) of the same device: $2.5 \mathrm{~K}$ (black), 3.5K (red), 4.5K (green), 6.5K (blue), 8.5K (cyan), 10.5K (pink). The left inset shows resistance versus temperature of a similar superconducting spin valve for the zero-field state (ZF), parallel (P), and antiparallel (AP) configurations $-\Delta T_{c}$ between $P$ and $A P$ is $810 \mathrm{mK}$. The right inset depicts a schematic diagram showing the dependence of $T_{c}$ on the angle between the ferromagnetic insulator surface spins ( $N$ : normal, S: superconducting) - the two horizontal lines illustrate the high and low temperature regimes discussed in the text.

The MR originates from the difference in $T_{c}$ between $\mathrm{P}$ and AP alignment (left inset to Fig. $1 \mathrm{~b}$ and Fig. 3d) and the appearance of an AP state over an extended field range between lower and upper coercive fields, $H_{c 1,2}$, corresponding to the switching of the $5 \mathrm{~nm}$ and $3 \mathrm{~nm}$ GdN layers respectively. This is evident in low $T$ magnetization vs field $(M(H))$ loops (Fig. 1b). For temperatures well above $T_{c}$, there is little evidence of separate switching of the two GdN layers despite their different thicknesses.

Fig. 2 shows the temperature dependence of $H_{c 1,2}$ for several $t_{S}$; in the normal state, $H_{c 1,2}$ are similar to each other and between samples. However, for each $t_{S}$, below $T_{c}, H_{c 2}$ increases rapidly with decreasing temperature suggesting the onset of a pronounced AF exchange coupling in the superconducting state. Our results provide the first experimental evidence for a superconducting exchange coupling (SEC), originally proposed by de Gennes ${ }^{10}$, mediated 




Figure 2| Influence of superconductor thickness on temperature-dependent coercive fields and spin valve effect in $\mathbf{G d N / N b / G d N ~ h e t e r o s t r u c t u r e s . ~ T e m p e r a t u r e - d e p e n d e n t ~ c o e r c i v e ~ f i e l d s ~ o f ~}$ $3 \mathrm{~nm}$ (solid symbols) and $5 \mathrm{~nm}$ (open symbols) GdN layers in SSVs for several $\mathrm{Nb}$ interlayer thicknesses: $8 \mathrm{~nm}$ (black), $10 \mathrm{~nm}$ (red), $12 \mathrm{~nm}$ (green) and $16 \mathrm{~nm}$ (blue) with data from $M(H)$ and $R(H)$ plotted as circles and squares respectively. The shaded regions represent the temperature between the onset of superconductivity (AP state) and the ending of superconducting transition ( $P$ state) in SSVs. The grey and dark grey curves show data for plain $5 \mathrm{~nm}$ and $3 \mathrm{~nm} \mathrm{GdN}$ films respectively. The left inset plots the difference in $T_{c}$ between the $\mathrm{P}$ and $\mathrm{AP}$ alignments as a function of Nb layer thickness. The right inset shows $R(T)$ curves for the devices plotted in the main figure (solid: AP, dashed P).

by the dependence of the superconductivity on the angle between the ferromagnetic insulator (FI) magnetizations in a SSV.

In order to distinguish this effect from other temperature-induced changes to $H_{c}$, we plot, in Fig. 2, $H_{c}$ from 5 and $3 \mathrm{~nm}$-thick plain GdN films (i.e. corresponding respectively to $H_{c 1}$ and $H_{c 2}$ in the SSV samples). The SSV $H_{c 2}$ data increase above the $3 \mathrm{~nm}$ trend line at the onset of superconductivity, as expected with an additional exchange term associated with superconductivity. At higher temperatures $H_{c 1}$ and $H_{c 2}$ differ less than the plain films, indicative of an underlying ferromagnetic Néel coupling mediated via stray fields originating from surface roughness.

Trilayer structures were also fabricated (in a single deposition run), with either a $\mathrm{Nb}$ or non-superconducting Ta interlayer between identical GdN layers (see Methods and Supplementary Information 2). Above the $T_{c}$ of $\mathrm{Nb} \mathrm{SSV}, H_{c 2}$ was identical in each case but, below its $T_{c}, H_{c 2}$ was significantly higher in the $\mathrm{Nb}$ sample, further confirming the existence of SEC as described above. Fig. 3a shows example hysteresis loops, whilst all $M(H)$ loops and a plot of $H_{c 1,2}$ versus temperature are shown in Supplementary Fig. 2 and 3.

We have also grown GdN/AlN/Nb/AlN/GdN control samples (see Methods), which show a much higher $T_{c}$ than their SSV equivalents and no response to the magnetic orientation, confirming that MR and $\Delta T_{c}$ are not a result of stray field. $\Delta T_{c}$ in a FI/S/FI SSV 
does originate from a net exchange field $\bar{h}$ acting on the singlet Cooper pairs gained during their reflection from the $\mathrm{S} / \mathrm{FI}$ interface. It was shown by de Gennes ${ }^{10}$, in the limit $t_{S} \ll \xi_{S}$, $\bar{h}=A \cos (\theta / 2)$, where $A$ depends on the interfacial spin coupling, $\theta$ is the angle between the surface spins of the FI layers, and $\xi_{S}$ is the S layer coherence length. In this limit, which is satisfied for $t_{S} \lesssim 12 \mathrm{~nm}$ in our experiments and for applied fields well below the critical field of the S, $\bar{h}$ and hence the $T_{c}$, are determined exclusively by $\theta$ (see sketched inset to Fig. $1 \mathrm{~b}$, Supplementary Information 3 and Supplementary Fig. 4).

The superconducting condensation energy per unit area is given by $E_{c}=t_{S} N(0) \Delta^{2} / 2$ where $N(0)$ is the density of states at the Fermi energy and $\Delta$ is the energy gap. In SSVs, because $T_{c}$ varies with $\theta, \Delta$ will also be angle-dependent and, using the usual expression valid near $T_{c}, \Delta(T, \theta) \simeq 3.2 k_{B} T_{c}(\theta) \sqrt{1-T / T_{c}(\theta)}$ where $k_{B}$ is Boltzmann's constant. In the regime where the SEC is directly measurable by the SSV effect the P configuration remains in the normal state and so the SEC energy $E_{e x S}$ (i.e. the difference in the condensation energy between the $\mathrm{P}$ and AP states) is given by


Figure 3 | Superconductor-mediated antiferromagnetic exchange coupling in GdN/Nb/GdN SSVs. a, $M(H)$ loops of a GdN(3)/Nb(8)/GdN(5) SSV and a GdN(3)/Ta(8)/GdN(5) control sample measured at 1.6K (red for Nb SSV and black for Ta control sample) and 4K (orange for Nb SSV and blue for Ta control sample). b, $M(H)$ loops of a $\mathrm{GdN}(3) / \mathrm{Nb}(7.6) / \mathrm{GdN}(5)$ (red) and a $\mathrm{GdN}(3) / \mathrm{Nb}(5.5) / \mathrm{GdN}(5)$ (black) trilayer measured at $1.6 \mathrm{~K}$. The inset illustrates $R(T)$ cooling (red) and heating (blue) curves of the same $\mathrm{GdN}(3) / \mathrm{Nb}(7.6) / \mathrm{GdN}(5)$ trilayer measured at 50mT after $\mathrm{P}$ alignments. The sample was first saturated using $150 \mathrm{mT}$ at $2.5 \mathrm{~K}$; the field was then reduced to $50 \mathrm{mT}$ and kept constant throughout the cooling sequence. Then the sample was saturated again at $0.3 \mathrm{~K}$ with the same procedure and warmed up. c, $R(H)$ of $\mathrm{GdN}(3) / \mathrm{Nb}(7.6) / \mathrm{GdN}(5)$ trilayer measured at three different temperatures. The arrows show the field sweep direction. $d, R(T)$ heating curves of the same $\mathrm{GdN}(3) / \mathrm{Nb}(7.6) / \mathrm{GdN}(5)$ trilayer measured at different external applied fields. These curves show AP alignment, zero-field alignment and increasing $P$ alignment in increasing field $(0->-80 \mathrm{mT}$ after saturation). 


$$
E_{\text {exS }}(T)=\Delta E_{c}(T)=\frac{3.2^{2}}{2} t_{S} N(0) k_{B}^{2} T_{c 0}^{2}\left(1-\frac{T}{T_{c 0}}\right)
$$

where $T_{c 0}$ is the critical temperature in the AP state. At the onset of superconductivity in the $\mathrm{P}$ state, $E_{e x S}\left(T_{c 0}-\Delta T_{c}\right) \simeq 1.5 \gamma t_{S} T_{c 0} \Delta T_{c}$ where the electronic heat constant $\gamma_{N b}=$ $\pi^{2} k_{B}{ }^{2} N(0) / 3=7.8 \mathrm{~mJ} \mathrm{~mol}^{-1} \mathrm{~K}^{-2}{ }^{18}$ and so for a SSV with $t_{S}=10 \mathrm{~nm}$, $E_{e x S}\left(T_{c 0}-\Delta T_{c}\right)=1.5 \times 10^{-5} \mathrm{~J} / \mathrm{m}^{2}$. For comparison, the Zeeman energy associated with the reversal at $H_{c 2}$ is given by $\Delta E_{Z}=2 \mu_{0} H_{c 2} t_{2} M_{2}$, where $t_{2}, M_{2}$ are the thickness and magnetization of the relevant GdN layer respectively. Using the magnetization data measured for these samples, for the $3 \mathrm{~nm}$ layer and a switching field of $5 \mathrm{mT}, \Delta E_{Z}=3.3 \times 10^{-5} \cdot \mathrm{J} / \mathrm{m}^{2} . E_{\text {exS }}$ is therefore comparable in magnitude to $\Delta E_{Z}$ and hence is large enough to control the magnetic switching since, in real magnetic structures, the Zeeman energy has to overcome the activation energies associated with anisotropy and domain wall pinning in addition to any exchange coupling. As shown in the left inset to Fig. $2 \Delta T_{c}$ increases dramatically with decreasing $t_{S}$ (Fig. $3 \mathrm{~d}$ shows a value of $>1700 \mathrm{mK}$ for $t_{S}=7.6 \mathrm{~nm}$ ).

Fig. $3 \mathrm{~b}, \mathrm{c}, \mathrm{d}$ show data for the thinnest superconducting sample $\left(t_{S}=7.6 \mathrm{~nm}\right)$ providing further evidence in support of this model. Fig. $3 \mathrm{~b}$ compares the $M(H)$ loop for this sample with a $t_{S}=5.5 \mathrm{~nm}$ sample which does not become superconducting down to $300 \mathrm{mK}$; the AP state originating from the SEC is present in the thicker sample but absent in the otherwise identical thinner sample.

In Fig. 3c, we can see that the transitions between the normal and superconducting states at $1.6 \mathrm{~K}$ are sharp and clearly driven by partial or complete bulk magnetization reversal of GdN layers between P and AP state as in Fig. 1a. SEC in this (higher) temperature range is reduced, and values of $\theta$ close to $\pi$ are necessary for superconductivity (see green line in the sketch inset to Fig. 1b). As the temperature is reduced, the normal to superconducting transition becomes broader. At the lowest temperature, this is also true for the superconducting to normal transition; at which point the transitions occur at virtually the same field magnitude. To explain this, we note that the GdN surface spins may be more weakly exchange coupled than the bulk of the film and so are able to reorient themselves under the action of the SEC. This is a similar picture to the "loose spins" model invoked by Slonczewski ${ }^{19}$ for metallic ferromagnetic films and is likely given that the interface is to a reactive metal $(\mathrm{Nb})$ and that $\mathrm{GdN}$ magnetism is strongly sensitive to nitrogen stoichiometry ${ }^{20}$. At lower temperatures much smaller values of $\theta$ are required to achieve a superconducting state (see grey line in sketch inset to Fig. 1b) and so the normal to superconducting transition can occur by the rotation of the surface spins away from the bulk GdN magnetization under the action of the AF SEC. Here there is a torque balance between the combination of the applied field and bulk exchange coupling to the $\sim \mathrm{P}$ aligned GdN layers, tending to align the surface spins in P state, versus the AF SEC which tends to drive the surface spins into AP state. Hence similar transition fields to and from the superconducting state are expected. The actual P-AP and AP-P bulk magnetization reversals take place at fields between the superconducting transitions and are therefore invisible from the low temperature $R(H)$ measurement.

Further evidence for this loose spins behaviour can be seen in Supplementary Fig. 4. The accuracy of the fit of the experimental data to the de Gennes model ${ }^{10}$ degrades as the 
nominally $\mathrm{P}$ state is approached and this can be explained most easily by the relaxation of the surface spins away from P under the action of the SEC so that substantial fields are required to fully align the spins to give the lowest resistive transition (see Fig. 3d).

The SEC discovered in our experiments is quite distinct from the blocking by superconductivity of a pre-existing normal-state indirect exchange coupling, originally proposed by Sipr and Gyorffy ${ }^{21}$ and by Sá de Melo ${ }^{22}$. This has been experimentally observed ${ }^{23}$; however, in complete contrast to the behaviour reported here, the pre-existing exchange interaction for the $\mathrm{GdN} / \mathrm{NbN} / \mathrm{GdN}$ studied in ${ }^{23}$ is $A F$ which is explained in terms of the semimetallic nature of the $\mathrm{NbN}$ spacer, and this vanishes in the superconducting state. For a metallic spacer such as $\mathrm{Nb}$ the RKKY interaction is much shorter-range than the values of $t_{S}$ studied here and oscillates between ferromagnetic and $\mathrm{AF}^{24}$ and so is unlikely to be relevant to our case. A localized ferromagnetic magnetostatic coupling between the FM layers modified by the appearance of superconductivity or by magnetostatic pinning of domain walls in the FMs by Abrikosov vortices in the $\mathrm{S}^{25}$ has recently been reported; however, this seems to involve modification of stray fields coupling the layers, rather than an intrinsic superconducting state controlled by the magnetic configuration and vice versa as reported here.

From our analysis it is evident the strength of the SEC depends on a number of superconductor parameters: $\mathrm{Nb}$ has one of the highest values of $\gamma$ and considerably higher than $\mathrm{Al}^{18}$ or $\mathrm{NbN}^{26}$ which are the most obvious alternatives; the product $t_{s} \Delta T_{c}$ depends strongly on $\xi_{S}$, which is likely to rule out $\mathrm{NbN}$ because of its much shorter coherence length than $\mathrm{Nb}$ (indeed no SSV effect was observed in GdN/NbN/GdN structures ${ }^{23}$ ), and is considerably higher than has been obtained for Al-based SSV results ${ }^{5}$. Finally, even though suppressed from its bulk value, $T_{c 0}$ for $\mathrm{Nb}$ is higher than for $\mathrm{Al}{ }^{5}$.

In contrast with our samples, softer FM layers may be expected to show complete AF SEC. In Fig. $3 \mathrm{~b}$ inset, the sample was saturated at $150 \mathrm{mT}$ at $2.5 \mathrm{~K}$ and $0.3 \mathrm{~K}$ before cooling and heating respectively; the field was reduced to $50 \mathrm{mT}$ throughout each measurement. The pronounced $R(T)$ hysteresis illustrates that, during the warming measurement, the sample remains in the superconducting state to a higher temperature, implying the existence of $\mathrm{AF}$ SEC and providing evidence that GdN layers are not soft enough to fully align in AP state during cooling. Softer FM layers will be switched completely by the SEC, which acts against the Néel coupling, leading to the observation of zero-field $T_{c}$ coincident with AP state $T_{c}$.

In conclusion, the behaviour reported here cannot be explained on the basis of any conventional exchange coupling. As importantly, unlike conventional exchange interactions, the SEC can be externally controlled by changing the superconducting state. Trivially this is achieved by varying the temperature as reported here, but the strong dependence of the energy gap on the quasiparticle density gives rise to large non-equilibrium effects.

Particularly, dramatic changes in the superconducting state in the central layer of a $\mathrm{S} / \mathrm{I} / \mathrm{S}^{\prime} / \mathrm{I} / \mathrm{S}$ double tunnel junction can be obtained if high transparency insulator (I) barriers are used ${ }^{27}$. GdN layers of comparable thickness to those reported here form good quality tunnel barriers ${ }^{28,29}$ and so the potential exists to create spin valve Josephson junctions ${ }^{8}$ in which the magnetic state can be changed by non-equilibrium current injection. Such devices are central to the current technological push for cryogenic memory ${ }^{9,30}$ and superspintronic devices ${ }^{11}$. 


\section{References}

1 Ryazanov, V. V. et al. Coupling of two superconductors through a ferromagnet: Evidence for a pi junction. Phys. Rev. Lett. 86, 2427-2430 (2001).

2 Robinson, J. W. A., Witt, J. D. S. \& Blamire, M. G. Controlled Injection of SpinTriplet Supercurrents into a Strong Ferromagnet. Science 329, 59-61 (2010).

3 Khaire, T. S., Khasawneh, M. A., Pratt, W. P. \& Birge, N. O. Observation of SpinTriplet Superconductivity in Co-Based Josephson Junctions. Phys. Rev. Lett. 104, 137002, doi:10.1103/PhysRevLett.104.137002 (2010).

4 Tagirov, L. R. Low-field superconducting spin switch based on a superconductor/ferromagnet multilayer. Phys. Rev. Lett. 83, 2058-2061 (1999).

5 Li, B. et al. Superconducting Spin Switch with Infinite Magnetoresistance Induced by an Internal Exchange Field. Phys. Rev. Lett. 110, 097001, doi:10.1103/PhysRevLett.110.097001 (2013).

6 Gu, Y., Halász, G. B., Robinson, J. W. A. \& Blamire, M. G. Large superconducting spin valve effect and ultra-small exchange-splitting in epitaxial rare-earth-niobium trilayers. Phys. Rev. Lett. 115, 067201 (2015).

$7 \mathrm{Gu}, \mathrm{J}$. Y. et al. Magnetization orientation dependence of the superconducting transition temperature in ferromagnet-superconductor-ferromagnet system: $\mathrm{CuNi} / \mathrm{Nb} / \mathrm{CuNi}$. Phys. Rev. Lett. 89, 267001 (2002).

8 Bell, C. et al. Controllable Josephson current through a pseudospin-valve structure. Appl. Phys. Lett. 84, 1153-1155 (2004).

9 Baek, B., Rippard, W. H., Benz, S. P., Russek, S. E. \& Dresselhaus, P. D. Hybrid superconducting-magnetic memory device using competing order parameters. Nature Commun. 5, 3888, doi:10.1038/ncomms4888 (2014).

10 de Gennes, P. G. Coupling between ferromagnets through a superconducting layer. Physics Letters 23, 10-11 (1966).

11 Robinson, J. W. A. \& Linder, J. Superconducting Spintronics. Nature Phys. 11, 307 315 (2015).

12 Katine, J. A., Albert, F. J., Buhrman, R. A., Myers, E. B. \& Ralph, D. C. Currentdriven magnetization reversal and spin-wave excitations in $\mathrm{Co} / \mathrm{Cu} / \mathrm{Co}$ pillars. Phys. Rev. Lett. 84, 3149-3152 (2000).

13 Meiklejohn, W. H. \& Bean, C. P. New magnetic anisotropy. Phys. Rev. 102, 14131414 (1956).

14 Bruno, P. \& Chappert, C. Oscillatory Coupling between Ferromagnetic Layers Separated by a Nonmagnetic Metal Spacer. Phys. Rev. Lett. 67, 1602-1605 (1991).

15 Baibich, M. N. et al. Giant magnetoresistance of (001) Fe $/(001) \mathrm{Cr}$ magnetic superlattices. Phys. Rev. Lett. 61, 2472-2475 (1988).

16 Grunberg, P., Schreiber, R., Pang, Y., Brodsky, M. B. \& Sowers, H. Layered Magnetic-Structures - Evidence For Antiferromagnetic Coupling Of Fe Layers Across Cr Interlayers. Phys. Rev. Lett. 57, 2442-2445 (1986).

17 Fullerton, E. E. et al. Antiferromagnetically coupled magnetic media layers for thermally stable high-density recording. Appl. Phys. Lett. 77, 3806-3808 (2000).

18 van Duzer, T. \& Turner, C. W. Principles of Superconductive Devices and Circuits. (Elsevier, 1981).

19 Slonczewski, J. C. Origin of biquadratic exchange in magnetic multilayers (invited). J. Appl. Phys. 73, 5957-5962 (1993).

20 Senapati, K., Fix, T., Vickers, M. E., Blamire, M. G. \& Barber, Z. H. Magnetic exchange hardening in polycrystalline GdN thin films. J. Phys.-Cond. Mat. 22, 302003, doi:10.1088/0953-8984/22/30/302003 (2010).

21 Sipr, O. \& Gyorffy, B. L. Oscillatory magnetic coupling between metallic multilayers across superconducting spacers. J Phys: Condens. Matter 7, 5239-5269 (1995). 
22 Sá de Melo, C. A. R. Magnetic exchange coupling in ferromagnet/superconductor/ferromagnet multilayers. Phys. Rev. Lett. 79, 1933-1936 (1997).

23 Senapati, K., Blamire, M. G. \& Barber, Z. H. Suppression of magnetic coupling in superconducting GdN-NbN-GdN trilayers. Appl. Phys. Lett. 103, 132406-132404, doi:10.1063/1.4822177 (2013).

24 Mattson, J. E., Sowers, C. H., Berger, A. \& Bader, S. D. Magnetoresistivity and oscillatory interlayer magnetic coupling of sputtered $\mathrm{Fe} / \mathrm{Nb}$ superlattices. Phys. Rev. Lett. 68, 3252-3255 (1992).

25 Curran, P. J. et al. Irreversible magnetization switching at the onset of superconductivity in a superconductor ferromagnet hybrid. Appl. Phys. Lett. 107, 262602, doi:10.1063/1.4938467 (2015).

26 Papaconstantopoulos, D. A., Pickett, W. E., Klein, B. M. \& Boyer, L. L. Electronic properties of transition-metal nitrides: The group- $\mathrm{V}$ and group-VI nitrides $\mathrm{VN}, \mathrm{NbN}$, TaN, CrN, MoN, and WN. Phys. Rev. B 31, 752-761 (1985).

27 Blamire, M. G., Kirk, E. C. G., Evetts, J. E. \& Klapwijk, T. M. Extreme CriticalTemperature Enhancement of $\mathrm{Al}$ by Tunneling in $\mathrm{Nb} / \mathrm{AlOx} / \mathrm{Al} / \mathrm{AlOx} / \mathrm{Nb}$ TunnelJunctions. Phys. Rev. Lett. 66, 220-223 (1991).

28 Senapati, K., Blamire, M. G. \& Barber, Z. H. Spin-filter Josephson junctions. Nature Mater. 10, 849-852, doi:10.1038/nmat3116 (2011).

29 Pal, A., Barber, Z. H., Robinson, J. W. A. \& Blamire, M. G. Pure second harmonic current-phase relation in spin-filter Josephson junctions. Nature Commun. 5, 3340, doi:10.1038/ncomms4340 (2014).

30 Gingrich, E. C. et al. Controllable $0-\pi$ Josephson junctions containing a ferromagnetic spin valve. Nature Phys. in press (2016).

\section{Acknowledgments}

We thank J.W.A. Robinson and B.J. Hickey for valuable discussions. This work was supported by ERC AdG "Superspin" and EPSRC Programme Grant EP/N017242/1.

\section{Author contributions}

Z.B. designed and coordinated the project, Y.Z. and A.P. designed and grew the samples and performed the measurements, M.B. developed the model. All authors contributed to writing the paper.

\section{Additional information}

Supplementary information is available in the online version of the paper. Reprints and permissions information is available online at www.nature.com/reprints. Correspondence and requests for materials should be addressed to M.B.

\section{Competing financial interests}

The authors declare no competing financial interests. 


\section{Methods}

Film growth. All films were grown on unheated oxidized single-crystal Si substrates by dc magnetron sputtering. The base pressure of the UHV chamber before sputtering was $\sim 5 \times 10^{-7} \mathrm{~Pa}$, with the background $\mathrm{O}_{2}$ and $\mathrm{H}_{2} \mathrm{O}$ partial pressure less than $2 \times 10^{-7} \mathrm{~Pa}$. Since $\mathrm{GdN}$ is highly reactive, low $\mathrm{O}_{2}$ and $\mathrm{H}_{2} \mathrm{O}$ partial pressure are crucial for obtaining good quality $\mathrm{GdN}$ films and sharp $\mathrm{GdN} / \mathrm{Nb}$ interfaces. The structure of our SSVs was $\operatorname{GdN}(3 \mathrm{~nm}) / \mathrm{Nb}\left(t_{S} \mathrm{~nm}\right) / \mathrm{GdN}(5 \mathrm{~nm})$. In order to protect $\mathrm{GdN}$ films, a $5 \mathrm{~nm}$ buffer and capping layer of AlN was deposited before and after the main SSV structure without breaking the vacuum. Substrates were placed on a computercontrolled rotating sample stage facing the $\mathrm{Gd}$ and $\mathrm{Nb}$ targets. To obtain a series of samples with different thicknesses in the same deposition run, substrates passed below the respective targets with varying rotation speeds. The GdN films were deposited in an $\mathrm{Ar} / \mathrm{N}_{2}$ gas atmosphere with $8 \% \mathrm{~N}_{2}$ at $1.5 \mathrm{~Pa}$, whereas the $\mathrm{Nb}$ films were prepared in pure Ar at 1.5 Pa. The AlN films, buffer, capping and spacer layers in control samples, were grown in an $\operatorname{Ar}(56 \%) / \mathrm{N}_{2}(44 \%)$ gas atmosphere at $1.25 \mathrm{~Pa}$. In addition, for the deposition of Ta control samples, the Ta films were prepared in pure $\mathrm{Ar}$ at $2.5 \mathrm{~Pa}$. The material parameters of plain $\mathrm{Nb}$ films are listed in Supplementary Table 1.

Magnetization measurements. Magnetization measurements were carried out down to $1.6 \mathrm{~K}$ using a vibrating sample magnetometer with a closed-cycle cryocooler. The magnetic field was applied parallel to the sample (in-plane); below $T_{c 0}$, very careful alignment of the sample plane with the magnetic field is required to prevent the $\mathrm{Nb}$ diamagnetism dominating $M(H)$ and so our $H_{c 1,2}$ data for lower temperatures are mainly extracted from the $R(H)$ loops.

Transport measurements. Transport measurements were performed on unpatterned $5 \times 5 \mathrm{~mm}$ samples using a four-point probe technique in a closed-cycle cryostat with a ${ }^{3} \mathrm{He}$ insert capable of reaching $0.3 \mathrm{~K}$. A constant bias-current of $10 \mu \mathrm{A}$ was applied in the measurements. During the isothermal measurements, the temperature stability of the cryostat was better than $10 \mathrm{mK}$. 